\title{
Coping with a fast-changing world: Towards new systems of future-oriented technology analysis
}

\author{
K. Matthias Weber ${ }^{1, *}$, Jennifer Cassingena Harper ${ }^{2}$, Totti Könnölä ${ }^{3}$ and \\ Vicente Carabias Barceló ${ }^{4}$ \\ ${ }^{1}$ AIT Austrian Institute of Technology, Department of Foresight \& Policy Development, \\ Donau-City-Straße 1, 1220 Vienna, Austria \\ ${ }^{2}$ Malta Council for Science and Technology, Villa Bighi, Bighi, Kalkara KKR 1320, Malta \\ ${ }^{3}$ Impetu Solutions, Victor Andrés Belaunde, 36-4C, 28036 Madrid, Spain \\ ${ }^{4} \mathrm{ZHAW}$ Zurich University of Applied Sciences, Institute of Sustainable Development, \\ Postfach, CH - 8401 Winterthur, Switzerland \\ *Corresponding author.Email: matthias.weber@ait.ac.at.
}

\begin{abstract}
Transformations linked to disruptive events are causing a shift in future-oriented technology analysis (FTA) activities from individual large-scale foresight actions to smaller in-house exercises and capacity building. The reasons are manifold relating to the need for an even tighter embedding of FTA in policy-making in a fast-changing complex environment as well as to internal drivers for novel forms of future intelligence to support coordinated and coherent decisions within and across organisations. This paper identifies three ideal types: external FTA services, the institutionalisation of FTA, and FTA networks, whilst recognising that in practice these types are complementary. In empirical terms this requires further investigation, in order to understand how different combinations of activities actually operate in their respective decision-making contexts. It is important to improve our understanding of how far institutionalised FTA can form part of customised solutions for building capacity to handle disruptions.
\end{abstract}

Keywords: future-oriented technology analysis; disruptions; institutionalisation.

\section{Introduction}

Increasingly dynamic processes of change and sudden disruptive developments are becoming the norm rather than the exception. The drivers of these changes may range from rapid technological changes to shifts in social norms, values and lifestyles. Disruptive events may stem from 'natural' to 'man-made' causes (financial and economic crises or social upheaval such as the Arab Spring). Similarly, in many respects, breakthrough technologies due to developments in information and communication technologies and nano- and biotechnologies have disruptive impacts on economies, markets and innovative consumer goods and services. Economies, socio-political and ecological systems are consequently under pressure to adapt, and undergo major structural and systemic shifts to new and more sustainable ways of innovating, producing and consuming (Könnölä et al. 2012a).

The need to transform current systems is heightened by emerging global trends, in particular democratic crises in many societies worldwide, the growing political and economic power of Brazil, Russia, India and China, the global reach of climate change and associated health and environmental risks. These call for new types of institutions that effectively design, implement and monitor joint action at international level (Amanatidou 2008; Brummer et al. 2008). Increased mobility, the instantaneous impact of events through social media and the sociocultural interconnections linking Europe to the rest of 
the world, lead to reverberations in European society. These developments heighten the uncertainty in the face of likely transformation processes ahead which call for strategic orientations (cf. Cagnin et al. 2012; Keenan et al. 2012).

Disruptive events highlight the vulnerability of governance and economic systems at local, national, European and global levels in the face of sudden crises and point to serious shortcomings in our ability to anticipate and prepare for disruptive developments. The dynamic international environment requires policy-makers to be better prepared for the 'unexpected' and to be in a position to instigate timely responses. New types of organisations and institutions are needed, which are sufficiently agile to transform their policies and practices in response to challenges. There is a need to enhance anticipatory, absorptive and adaptive capabilities and capacities in society to address continuous as well as disruptive change and challenges (Könnölä et al. 2012a). Current practices of FTA require transformation through effective embedding of FTA in a user context of governance structures and processes at corporate and government levels.

In recent years, institutional responses have included the setting up of horizon-scanning centres and similar anticipatory structures (Amanatidou et al. 2012; Van Rij 2010) together with the downscaling of national exercises and the embedding of FTA functions in organisations and their respective decision-making structures and processes. These responses entail certain risks and pitfalls. In order to conduct a systematic analysis of the strengths and weaknesses of different organisational models of FTA, an appropriate conceptual framework needs to be developed. We propose an approach which frames the wider societal requirements for FTA within specific forms of FTA systems, i.e. configurations of different elements required to deliver forward-looking intelligence in support of decision-making.

FTA systems cover three levels of elements and the interdependencies between them, namely:

- the individual capabilities and mindsets to anticipate change in fast-changing environments;

- the institutional and organisational set-up of FTA;

- the institutional context and modes of governance in which FTA is embedded.

In order to focus on the organisational and institutional aspects of FTA systems, the individual level of analysis will not be addressed in this paper. This simplified conceptual frame allows consideration of variants of FTA and their effectiveness in tackling a range of futures and related needs. A typology of future requirements to be addressed by FTA, including exploration of the future and preparedness for the unknown, will be developed. Drawing on recent experiences with alternative models of FTA systems, solutions will be identified based on a combination of social, organisational and technological innovations at the three levels of FTA systems, to make FTA fit for the challenges of the future.

Against this conceptual background the four guiding questions addressed in the subsequent sections are:

- What areas and types of transformations will require anticipatory action? And what kinds of requirements for FTA result from these?

- What kinds of models for FTA systems exist? How can they be systematised in conceptual terms?

- What kinds of developments can we observe in terms of how these models are used in practice?

- What do these findings suggest with regard to the future direction to take for organising FTA activities?

The structure of the remainder of this paper is as follows: Section 2 addresses the first two questions, including also the future conceptual requirements for FTA systems that are likely to emerge, and the types of organisational models and governance contexts that make up FTA systems. Section 3 will draw primarily on recent empirical research presented at the FTA 2011 Conference, ${ }^{1}$ which will be analysed using the dimensions and categories of the conceptual framework in order to identify emerging patterns and issues. The analysis will clarify the potential of different institutional models for tackling different types of future requirements. Section 4 brings together the analytical and normative perspectives and defines forward directions for coping institutionally with fast and disruptive change. It also points to key conceptual conclusions.

\section{Conceptual framework: Capturing new types of FTA systems in the light of specific circumstances}

This section introduces the conceptual framework for subsequent analysis of recent empirical research on FTA systems. Different types of contextual transformations that pose challenges for FTA systems are identified, taking into account the wider institutional setting and co-evolution of different governance modes. Building on three dimensions (transformation types, governance modes, and organisational models of FTA) a conceptual frame is developed for analysis in the empirical part of this paper.

\subsection{The fast-changing context: Requiring systemic and structural transformations}

The global context represents an arena of major disruptions triggered by natural and man-made events which are transforming our society and/or requiring transformation of our society, in highly significant ways. In this section, a typology of transformations is developed to distinguish between different forms and sources of change in the system. Whilst sudden and shocking 'black swan' events 
(Taleb 2007) dominate our attention, these can overshadow less noticeable and gradual processes of transformation which can have equally significant medium- to long-term impacts (consider the convergence of technologies, as discussed by Nordmann (2004)). The more impressive transformations are those which happen by design and are engineered through deliberate and concerted action, either gradually (transitions) (e.g. Rotmans et al. 2001; Geels and Schot 2007) or rapidly (policy shocks) (e.g. Skoufias 2003).

The categories of grand challenges identified by theEuropean Research Area (ERA) Rationales Expert Group (European Commission 2008) provide one typology of transformations. Economic challenges correspond to the agenda set out by the Aho Group (European Commission 2006) and call for a combination of supply-side and demand-side measures to create innovation-friendly markets. Social and environmental challenges deal with the causes and consequences of issues such as climate change, food and energy security and the ageing society, which require an initial drive from governments. Science and technology are also the basis of challenges involving the collective ability to respond to opportunities in frontier research. Different types of grand challenges call for different transformation models and policy strategies. The distinction between disruptive and recognised grand challenges referred to in the European Science Foundation report (European Science Foundation 2010) highlights the fact that areas of disruptive grand challenges can be exogenous or endogenous and are low-probability (emerging), high-impact issues that challenge societal and economic health. Recognised grand challenge areas are 'grand' in the sense that they are instantly recognisable as representing a major aspect of human or social well-being and prosperity and can be both a threat and an opportunity.

The typology adopted in this paper distinguishes between four broad types of transformations (see Fig. 1). First, disruptive events are unexpected, unprepared for, short-term and sudden but with immediate and ongoing impact(s). Secondly, ongoing processes of transformation change may be difficult to detect due to the gradual nature of the process and the medium- to long-term impacts associated with them, for example in the case of climate change or environmental change. Thirdly, two types of

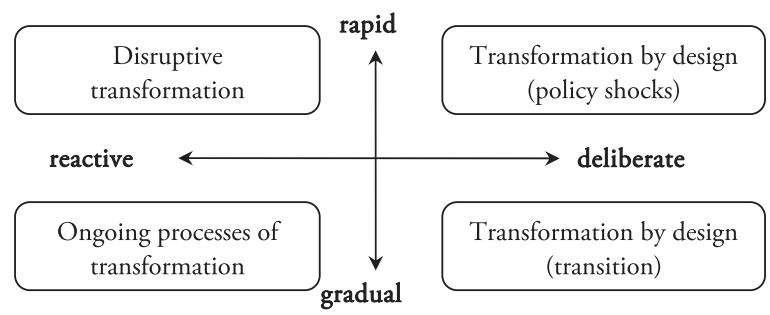

Figure 1. Identified types of transformations. transformation can come into effect by design where change processes are planned and implemented, for example economic structural transformation or social change. Depending on whether the transformations by design are gradual or rather abrupt, we speak of transitions or policy shocks.

The axes (see Fig. 1) highlight the divide between the primarily reactive approach to disruptive events and ongoing processes of transformation and the need to use FTA to move towards more deliberate, proactive approaches to transformation.

Each type of transformation requires a dedicated response drawing on particular knowledge and competencies. Transformations involve a complex, interactive chain of changes ranging from technological, natural, economic and political to social (pervasive and quick to diffuse with longer term effects emerging over time) and give rise to particular research needs. In the context of this intricate web of disruptive, natural and planned transformations FTA has a critical role to play in defining effective policy responses. These include; improving the quality and robustness of anticipatory intelligence and preparedness for disruptive events through systematic approaches and shared insights and perceptions; creating an effective dialogue between key players in the different policy domains; vision-building and consensus-building for engineering major processes of transformation and efforts to define the research agenda, setting research priorities and specialisation focus.

Disruptive events in recent years have made apparent critical weaknesses in the capacity to anticipate, prepare for and address comprehensively sudden events and the impacts they generate immediately and in deeper, more pervasive, but less obvious ways over time (Könnölä et al. 2012a). At the same time, the capacity to engineer and prepare for major breakthroughs resulting from scientific discovery, is an important driver of innovation and competitiveness, often requiring structural and systemic change and, in turn, necessitating the capacity to design and implement these transformations. Limitations to in-house capacity exist in institutions at national and international level and are compounded by weak collaborative links between these entities in sharing information (anticipatory intelligence and early warning), expertise and other resources required for effective policy responses.

\subsection{FTA systems and modes of governance}

FTA systems form an active part of the institutional context and governance structures managing systemic and structural transformations. This relationship with the governance context demarcates how FTA systems evolve and drive the anticipatory capabilities in society. Könnölä et al. (2009) derive four modes of governance from arguments that build on cultural theory (Tukker and Butter 


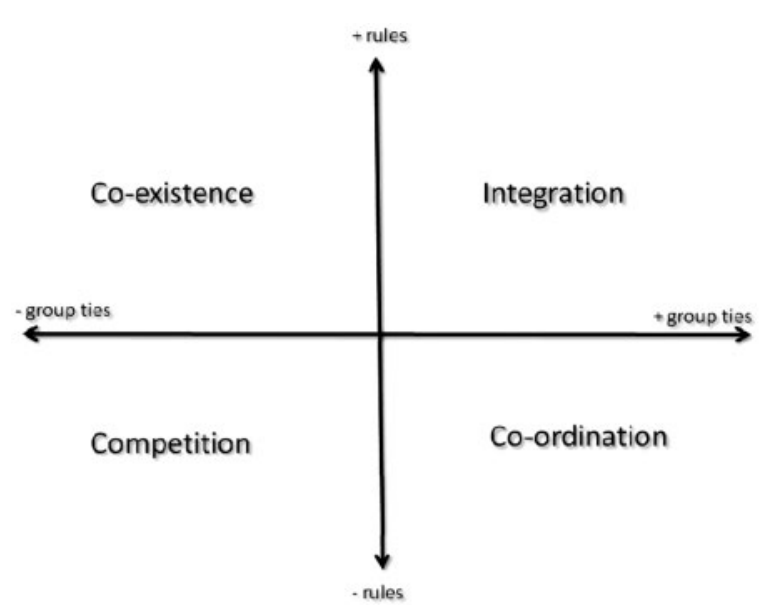

Figure 2. Modes of governance (modified from Thompson et al. 1990).

2007). Social organisation can be understood in terms of the extent to which an individual is bound in a unit or social group and the degree to which an individual's life is determined by external prescriptions or rules and norms (Thompson et al.1990). In our analysis these basic 'forces' are transposed to the societal (governance) level, allowing a deeper understanding of how governance exercises influence in the system. Two dimensions (rules and group ties) are used as axes to illustrate four approaches ${ }^{2}$ to social organisation.

Two dimensions, namely 'extent of group ties' and 'extent of influence of external rules' are used to construct a matrix that defines four modes of governance (see Fig. 2 and Table 1).

Further to such archetypal modes of governance, in practice we observe shifts in the governance structures in the course of societal transformations. For instance, transformations may emerge through proactive coordination that may lead to changes in competition and integration modes of governance. Alternatively, the governance system may adopt a co-existence mode until abrupt changes such as an economic crisis, force government to take up new measures in other modes of governance- for instance policy measures to incentivise research and innovation as a means of generating economic growth.

FTA systems both affect and are affected by shifts in governance modes. The integration mode is likely to lead to the implementation of normative approaches that support common vision-building and priority setting. In turn, for instance, the co-operation mode may pinpoint enhanced networking activities. Such dynamics call for further research into the design of tailored FTA systems which are fit for purpose.

\subsection{FTA systems and organisational models of FTA}

In defining our conceptual framework, three ideal-type organisational models for FTA can be identified, taking into account the speed of change and types of responses generated, namely:

- individual FTA projects or programmes of limited duration and with targeted objectives;

- dedicated FTA units providing continuous input to their embedding or mother organisations;

- FTA networks as informal yet stable settings allowing the bundling or coordination of resources and competencies.

2.3.1 Projects and programmes as external FTA services. Setting up dedicated and temporary FTA projects or programmes has been a very common model over the past two decades. Driven by the need to explore certain technological, economic or societal developments of major concern to decision-makers, FTA activities are largely geared to deliver specific responses to specific questions. Most national Delphi (cf. Cuhls 2001; Joos et al. 1999) and foresight processes in general follow this type of model (Butter et al. 2009). There are obvious exceptions to this rule and more recently there has been a growing trend to use foresight to open up broader and exploratory debates, involving the public as well as experts (cf. Loveridge and Street 2005; Könnölä et al. 2012a). A key issue related to specific programmes and projects concerns the ability to absorb the results into decision-making processes. Due to the typical customerclient relationship, the opportunities for joint learning and exploration are limited. The rationales, time horizons and capabilities of the clients and users determine to a large extent what types of results are taken up or not. Non-conventional irritating results tend to be ignored. To be effective, projects and programmes require a well-developed anticipatory capacity within the contracting organisations; a condition that is not always met. Disruptive trends, events and scenarios explored in programmes and projects and related findings, often prove difficult to transfer to potential users. This may be due to the fact that they regard themselves as clients and are not willing to become engaged in joint learning processes with open and unexpected outcomes.

Programmes and projects usually require access to extensive FTA expertise outside the client organisation. A landscape of research and consulting organisations therefore needs to be in place to deliver the services required. In other words, if the institutionalisation of FTA on the user side is low, a well-developed set of specialised organisations must be in place on the supplier side to generate FTA results.

2.3.2 Institutionalisation of FTA. There is an emerging stream of FTA activity geared to providing future-oriented knowledge for decision-making on a more continuous 
Table 1. Key characteristics of modes of governance

Integration mode of governance

- Proactive use of hierarchical structures with power and means to implement selected activities

- Transformations can be managed

- Institutionalised FTA supports the process of defining the vision and the route

Co-ordination mode of governance

- Coordination of voluntary engagement in coalitions in order to develop common activities

- Joint visions and action plans prepare for common futures

- Networked FTA

Competition mode of governance

- Optimising the market conditions

- Competing visions and action plans to cope with transformations

- Fragmented FTA

Co-existence mode of governance

- Reactive, wait-and-see until new opportunities

- Focus on resilience, not on visions

- Reduced FTA

basis through more institutionalised setups. This is reflected in the setting up of dedicated horizon-scanning centres (cf. Amanatidou et al. 2012) in the UK, Netherlands and Singapore at national or regional levels, aimed at identifying not only major long-term trends and drivers, but also possible disruptive events or developments that may radically influence the future. This trend reflects growing attention to uncertainties and complexities, and the need for faster delivery of FTA results to policy and decisionmaking. The rediscovery of parliamentary technology assessment (TA) is also a sign of renewed interest in institutionalised forms of TA (cf. Salo and Kuusi 2001).

The institutionalisation of FTA provides continuity and permits the accumulation of knowledge and know-how in a single organisational unit. Institutionalisation can take place in different forms, with varied degrees of centralisation and distribution, providing both integration and networking within and across organisations. The boundaries between service provision and institutionalisation can also be blurred, for instance in the case of external FTA organisation that provide semi-institutionalised FTA services (e.g. parliamentary TA organisations, such as the Scientific Technology Options Assessment Unit of the European Parliament, build on long-term service contracts with external public and private research organisations or consulting firms). However, in principle institutionalised FTA has the advantage of being firmly embedded further in policy and decision-making: it can draw on a close and trustworthy relationship with decision-makers and gives access to insider knowledge of the embedding organisation that allows them to bring their FTA insights fully to bear and help shift organisations towards new directions. Moreover, due to their stability, they can build up a stock of knowledge, including potential emergency plans for possible disruptions that may not be of immediate relevance, but could provide a fast-response capability which would be crucial in times of crisis. An advantage of FTA institutions is their visibility, rendering them attractive as network nodes in an international context.

Institutionalisation also has its drawbacks as organisations within a public administration may be at risk of ossifying. Too close relationships with the potential users in private or public organisations may inhibit the critical function that FTA should have (a problem shared with external FTA service providers who depend strongly on their clients). Embedding and institutionalisation thus seems to be most effective if coupled with some degree of autonomy in order to maintain a critical distance. This is crucial if they are to be able to point to future risks and potential disruptions that are not yet at the top of the current policy agendas.

2.3.3 FTA networks. A third model, more accessible to countries and organisations with limited resources, is the network model which has grown over the past decade. In FTA, networks can relate to individuals, and are peer-to-peer enabled through information technology and the internet (cf. Cachia et al. 2007). Examples of web 2.0 networks include: EFP, FORwiki, iKnow, and the Millenium project. ETEPS, EPTA Network, Eurasian Virtual Centre, Network TA in Germany, Austria and Switzerland are among the more conventional networks. As an organisational model, networks are complementary to FTA institutions and programmes/projects, interconnecting FTA knowledge hubs and providing smaller organisations with limited FTA capacity access to a wider pool of knowledge. In providing support on policy options and consequences in the face of disruptions, they have the potential to mobilise a distributed capacity in a flexible manner. However, most networks do not have the necessary processes in place to go beyond information exchange and occasional joint initiatives. They also require a minimum level of capacity and competence to be in place, as well as organisations - either institutionalised 
FTA units or research and consulting units - that can serve as network nodes. As a joint infrastructure, networks are of major benefit in facilitating the exchange of experiences, setting up training activities and communities of practice. Networks have a strong potential to enhance the visibility and coherence of FTA, but there have been only very few cases of networks being able to tackle the consequences of, and strategies for, handling disruptive and structural transformations.

\subsection{Framework for analysis of FTA systems}

The three dimensions introduced in the previous sections constitute the conceptual framework for analysing the suitability of different types of FTA systems in terms of their ability to tackle different types of transformative changes (see Table 2). The first dimension captures four types of transformations, which raise quite different requirements for FTA systems as defined by the two other dimensions of our framework. Institutions, networks and external services are regarded as the main organisational models for implementing FTA and constitute the second main dimension. However, which of these three basic organisational models best fits the requirements is also a matter of the governance mode (co-existence, competition, cooperation or integration) which constitutes the third dimension of the framework. In order to be able to respond to a transformation, organisational model and governance mode need to be compatible with each other.

\section{Diversity of FTA systems in practice}

Against this backdrop, it is important to develop a better understanding of how FTA can provide a solution to building an anticipatory capacity in a climate of high societal uncertainty and complexity. Towards this end, we examine in Section 3.1 how recent empirical research papers, including those presented at the FTA 2011 Conference, address the challenge of transformation, FTA organisation and governance modes, with the aim of teasing out the main trends in how FTA is evolving in practice. In Section 3.2 we focus on the interplay between these dimensions, explaining why the observed trends are plausible and thus provide an indication of how FTA might further evolve if the challenge of transformations persists.
From a historical perspective the recent debates on the need to tackle transformations and disruptive developments represent the most recent shift in the evolution of FTA. With regard to the rationales underlying the evolution of FTA, three main stages can be distinguished. First, the traditional and best established forms of FTA focus on the anticipation of rather gradual change that may require adjustments of how innovation systems are 'wired up' and what thematic, often science and technology, priorities should be addressed in these. FTA along these lines was in need of updates at regular intervals of three to five years, implying that individual projects or programmes were the most suitable form for addressing FTA tasks.

More recently, the scope of the problems and issues to be tackled by FTA has been expanding. Broader socio-economic questions have complemented scientifictechnological ones, but the focus of attention has remained on research and innovation (R\&I) policies, with greater attention being paid to the perspectives of societal stakeholders. Projects and programmes continued to be the dominant form of implementing FTA, complemented by the need to enhance networking to access knowledge in other domains of society.

What we can see emerging against the background of a new rationale for FTA, namely to address the entire R\&I ecology and its embedding in other policies to tackle grand societal challenges, is a change in both governance and organisational models. A much higher degree of policy coordination seems to be needed to address societal challenges as well as a much more continuous and 'embedded' approach to FTA. This is actually the main hypotheses we would like to propose in this paper. By looking into the latest developments in how FTA systems - understood as combination of governance modes and organisational models of FTA - have evolved in practice and allow responding to novel challenges (see Table 3), we want to explore the direction in which FTA is likely to evolve in the future.

\subsection{Emerging developments in FTA systems}

This section looks at how emerging developments in FTA deal with challenges of transformative change, by analysing recent contributions to the literature as presented at the 2011 FTA Conference. The analysis identifies the types of transformations addressed, the governance modes in which FTA is embedded and the organisational

Table 2. Framework for analysis of FTA systems

\begin{tabular}{llll}
\hline Dimensions & Transformation types & Organisational models of FTA & Governance modes \\
\hline Sub-categories & $\bullet$ Disruptive & $\bullet$ External services (projects and programmes) & $\bullet$ Co-existence \\
& $\bullet$ On-going processes & $\bullet$ Networks & $\bullet$ Competition \\
& $\bullet$ Gradual by design & $\bullet$ Institutionalisation & $\bullet$ Integration \\
\hline
\end{tabular}


Table 3. Changing rationales for FTA

Approaches on FTA systems

\begin{tabular}{|c|c|c|c|}
\hline \multirow[b]{2}{*}{ Dimensions } & & & \\
\hline & $\begin{array}{l}\text { Transformation types and } \\
\text { consequent challenges }\end{array}$ & Governance modes & Organisational models of FTA \\
\hline Traditional rationales for FTA & $\begin{array}{l}\text { - Anticipate gradual changes } \\
\text { and support deliberate policies } \\
\text { for transitions } \\
\text { - 'Wiring up the innovation } \\
\text { system' } \\
\text { - Stimulating national and } \\
\text { regional economic development } \\
\text { through innovation }\end{array}$ & $\begin{array}{l}\text { - Guiding and/or complement- } \\
\text { ing competition and integra- } \\
\text { tion oriented governance } \\
\text { - Designing research policy and } \\
\text { strategy with broad aim of } \\
\text { selecting priorities for research } \\
\text { investments and cooperation of } \\
\text { R\&I actors }\end{array}$ & - Programmes and projects \\
\hline Recent rationales for FTA & $\begin{array}{l}\text { Early identification of } \\
\text { emerging issues and conse- } \\
\text { quent trends and transitions in } \\
\text { society } \\
\text { - Interconnecting different areas } \\
\text { of research }\end{array}$ & $\begin{array}{l}\text { - Enhancing coordination in } \\
\text { research through FTA } \\
\text { - Evidence-based, joined-up R\&I } \\
\text { policy } \\
\text { - Consensus-building and effect- } \\
\text { ive engagement of civil society }\end{array}$ & $\begin{array}{l}\text { - Programmes and projects } \\
\text { - Networking to address open } \\
\text { innovation }\end{array}$ \\
\hline
\end{tabular}

models that are applied. While the number of papers selected for analysis is limited, the FTA conferences ${ }^{3}$ represent focal points for addressing issues of how FTA is embedded in governance processes, and are thus a good indicator of ongoing developments in FTA. The analysis of the selected papers on FTA attests to the richness of approaches in relation to different types of transformations, governance modes and consequent models on organising FTA activities (see Table 4).

Our analysis of the selected papers indicates an increasing emphasis in FTA objectives on improved understanding of transformations. Furthermore, there seems to be considerable richness of foci to different types of transformations. In a number of exercises the methods aim to explore and understand highly complex and often uncertain future developments by way of addressing, in the same exercise, alternative forms of transformation and the consequent roles of different stakeholders. In this context, scenario work seems to allow fairly flexible frames for such overarching discussions, whereas a number of other methods are applied to develop future plans and action-oriented recommendations for decision-making. It should be noted that while several exercises address disruptive transformations, only few remarks are made on consequent organisational responses to extreme uncertainty, for instance by way of resilience measures.

In terms of addressing the governance modes, the papers indicate particular attention to the coordination mode, reflecting the theoretical premises of prevailing foresight practice with its emphasis on systemic aspects and networking. Papers with a strong emphasis on the innovation system, tend to also address other dimensions and their interplay with the coordination mode. This indicates that FTA has potential to support 'policy mix' (demand- and supply-side) approaches and the comprehensive analysis of possible measures in view of their combined effects on different governance modes. Some papers are interestingly addressing the systemic qualities of the context in which FTA is conducted by way of exploring what happens in the innovation system before and after FTA is conducted. Rijkens-Klomp and van der Duin (2012) have illustrated that when a governmental organisation decides to use FTA methods, they experience it as a big step, often even a step of transformation of the organisational culture, a new way of thinking and acting throughout the organisation, because routines in strategic policy processes do not always match with the characteristics of required methods.

In a search for effective organisational forms of FTA systems, the papers presented at the 2011 FTA Conference call for a stock-taking of diverse forms of FTA activities, and for establishing FTA functions in the organisations or innovation systems compatible with the underpinning culture, institutional conditions and available capacities and resources. Such observations lead to diverse forms of organisational setups for FTA. Along similar lines, Weber et al. (2011) have analysed types of 
Table 4. Diversity of FTA systems in practice

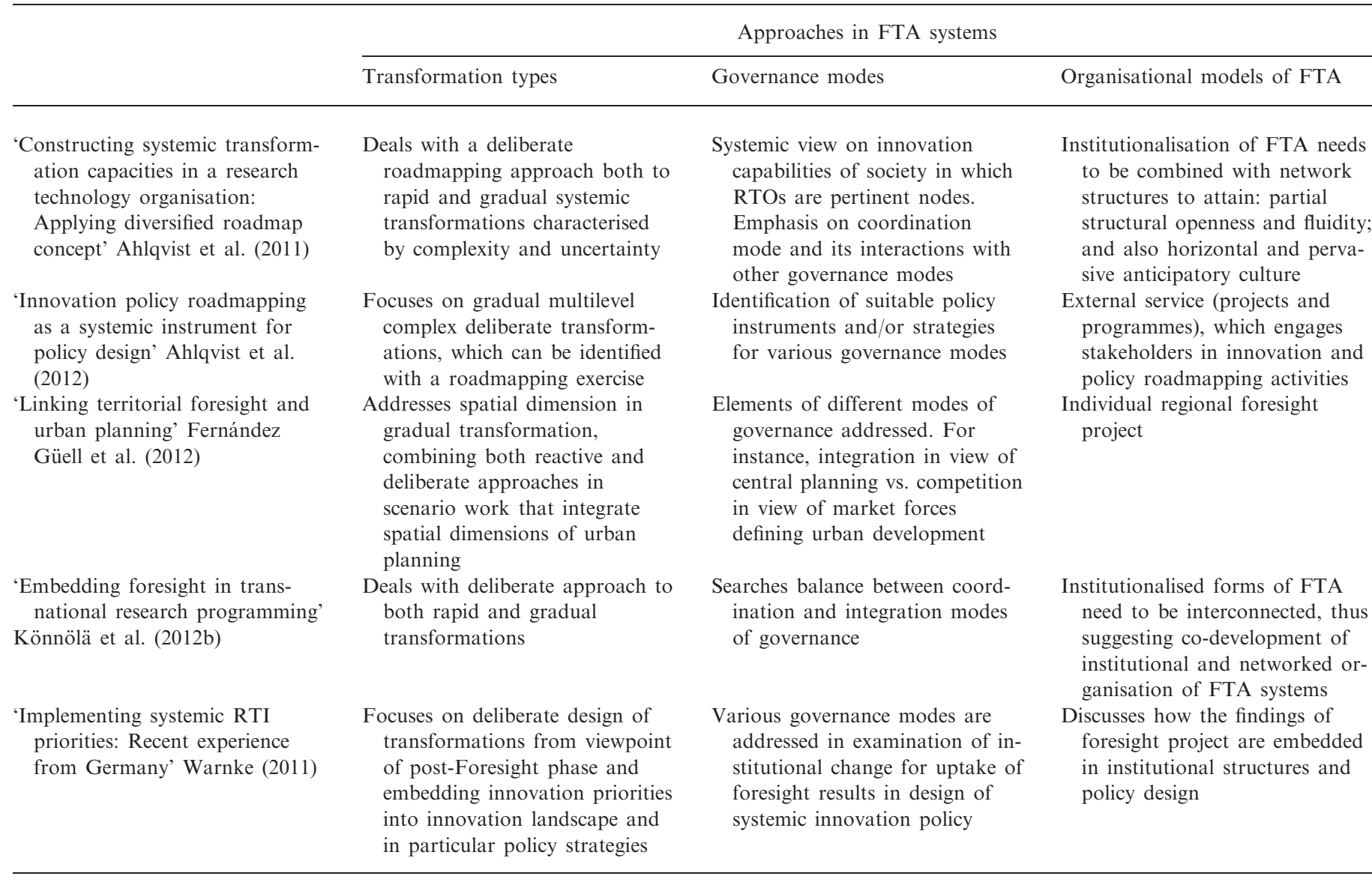

forward-looking or foresight activities tailored to exploring the future in the context of science, technology and innovation (STI) policies and achieving impact on national innovation systems (NIS). They argue that external FTA services are useful if there is a clear objective of the service, such as helping define priorities, or suggesting future fields in a specific phase of re-orientation of policy, whereas institutionalised forms of FTA are more suitable under unstable political conditions and in situations with a high level of fluctuation.

The majority of the paper presented at the 2011 FTA Conference, despite reporting on experiences derived from a single foresight exercise, indicate increasing evidence of institutionalised forms of FTA. The institutionalisation of FTA seems to generate intense discussion on the benefits and drawbacks of stronger integration in the policy processes. For instance, there is a risk of cooptation of incumbent positions rather than exploring novel alternatives for decision-making. In particular Warnke (2011) recommends the use of strategic dialogues to foster the embedding of suggested 'future fields' into the national research, technology and innovation (RTI) landscape. Ahlqvist et al. (2011) outline paths to enable anticipatory culture in research and technology organisations
(RTOs) and other organisations. They suggest changing this anticipatory thinking by transforming the foresight process into a horizontal organisational function that permeates all levels of the parallel innovation process. In another example of embedded FTA, Calof (2012) as well as Calof et al. (2012) report on the setting up of foresight units within several departments at the federal level in Canada. In essence, we can conclude that the horizontal convergence of FTA competencies is what is called for, in order to help FTA move closer to decision-making. As a consequence, the absorptive capacity in decision-making bodies needs to be enhanced, calling for a change in organisational culture.

Networks of FTA practice generate a range of positive impacts particularly in engaging external, often international, experts in FTA. Nehme et al. (2011) address the importance of engaging stakeholders in foresight exercises from the very beginning in order to improve support for policy options and their implications. We can conclude that a substantial benefit can be derived from such networks, in particular for smaller countries or countries with a less developed foresight and anticipatory intelligence culture in general. Tiits and Kalvet (2011) learned from recent foresight exercises in Estonia that the 
'intelligent piggybacking' approach is much more suitable for smaller catching-up economies than the traditional 'grand narratives' approach typically employed in larger advanced economies to define future developments at the cutting edge of a given field of technology. Moreover, they conclude that foresight exercises carried out in smaller countries require international support. International cooperation, in particular when assisted by FTA networks, can raise awareness among policy-makers and other stakeholders and help to validate foresight processes and the results that they generate.

To conclude, the alignment of FTA with decisionmaking and the coordination mode of governance prevail in recent FTA despite the considerable richness of foci on different types of transformations and methodological choices and organisational setups for FTA. Furthermore, our analysis shows increasing evidence of institutionalised forms of FTA and exploitation of FTA networks to provide agile and strategic support for decision-making.

\subsection{Reflecting on the way forward with FTA systems}

The above review of recent research work indicates a considerable diversity of FTA approaches and systems with co-existing diverse forms and types of transformations, modes of governance and organisational models. A number of cross-cutting observations can be drawn on the current evolution of FTA, on emerging requirements and possible responses to them.

\subsubsection{Observation 1: Emerging requirement profiles} for FTA systems. The increasingly dynamic global and national context implies a greater breadth and depth of functions to be provided by FTA systems, which in turn requires tailored approaches and highly specific skills. Embedded and networked FTA facilities are particularly suitable to fulfil the following functions for innovation policy ${ }^{4}$ in such a dynamic context:

- Early warning of disruptive events and scientific/ technological breakthrough and their likely impacts in scope and time. In order to realize this function, horizon-scanning centres or units at national, regional and international level are emerging. These carry out their work in open networked mode, sharing information at international level with their counterparts in other countries and also internally within the country, with government, business, academia and the public. This networked mode of operations links with social networks as a means for identifying weak signals. ${ }^{5}$

- Addressing deficiencies or shortfalls in the R\&I ecology relating to lock-in to obsolete technologies or business models, and old networks which require realignment.
This can address bottlenecks in the framework conditions hampering the whole process from ideas to innovation. ${ }^{6}$ Smart specialisation and the contextualisation of R\&I policy mix are emerging priorities. Local, regional and national FTA networks, cutting across different policy areas, can support an appropriate level of policy coordination to anticipate and tackle structural deficiencies in the R\&I ecology.

- Addressing grand challenges requires public appreciation of the important role played by R\&I in meeting social, environmental and other needs. Grand challenges go beyond the domain of research policy and require structured interactions with a broad range of related policy domains (European Research Area Board 2009; Weber and Georghiou 2010). They also depend on governments and international institutions working together to define common research agendas. Worldwide and regional (European) networks of the FTA units in international organisations, national and-at least for some grand challenges - regional (subnational) governments, business and nongovernmental organisations are providing an organisational solution to share policy perspectives and develop joint policy and research agenda.

The subsequent observations deal with the question of how these requirements and challenges can be addressed by combinations of governance contexts and appropriate organisational models of FTA.

\subsubsection{Observation 2: The need to strengthen} networking. The need to handle increasingly global challenges requires accessing a broad spectrum of sources of knowledge for anticipating transformations as well as possible solutions. Networked approaches to FTA have been growing in importance for several years, and this trend is likely to continue as part of a comprehensive package of elements for dealing with transformative change, including a more widespread use of web 2.0 tools.

In addition to access to new resources and knowledge, Könnölä et al (2012a) consider that international networking and easy access to a wider set of stakeholders is important to make sense of emerging issues. While individual and organisational sense-making has been aptly recognised as a key determinant of what signals are 'relevant', the collective inter-organisational sense-making processes coupled with the interpretation of signals have received less attention, particularly as concerns the recognition of interconnections among the signals or the derivation of their policy implications (Dervin 1998). These collective processes are important because they underlie key scoping decisions on scanning emerging issues and thus shape both implicit and explicit expectations that influence scanners and their behaviour. Overall, networks for the collaborative development of cross-cutting challenges 
may help reframe the 'bigger picture' whose exploration paves the way for policy coordination and the attainment of systemic policy objectives.

\subsubsection{Observation 3: Institutionalisation in response} to changing requirements. In recent years, there has been a growing tendency among governments and businesses to invest less in individual large-scale foresight programmes and projects, and to opt more for developing in-house competencies for coping with sudden change. A shift towards institutionalisation of FTA could be observed, as reflected, for instance, in the creation of new dedicated horizon-scanning centres, the strengthening of parliamentary technology assessment offices and the establishment of dedicated foresight units in firms and public administration.

There are many reasons for this move from projects and programmes to institutionalised forms of FTA. They are due both to the more volatile and uncertain context for policy-making and to the growing demands for transparency and accountability. In many policy areas, both public and private sector, a strategic perspective on decisionmaking is necessary in order to handle limited resources. While this strategic orientation may be common in the private sector, it is a rather new development in the public sector, for instance in the field of RTI policy, where matters of priority setting have acquired greater prominence (Georghiou and Harper 2011). Decisionmaking in organisations themselves has become more complex, and the level of coordination needed to achieve coherent decisions adds to this. Institutionalised FTA capacities are required to support this type of coordination.

As a consequence, there is a growing need to embed this anticipatory capacity in policy and decision-making quickly, strategically and on a continuous basis. This is critical not only to be able to respond and adapt to new situations before they occur, but also to shape the future, building upon mutual understanding and common visions which are to be jointly pursued. Embedding FTA in organisations is regarded as a vehicle to make them more anticipatory and adaptive to enable a continuous, systemic and structural transformation of organisations' premises and practices, with the ultimate goal of handling current and future technological, economic and societal challenges in line with the goals defined by the organisation.

In the case of parliamentary TA, the argument in favour of dedicated future intelligence can be made in a very straightforward manner. In general, parliamentarians need better access to knowledge about current and future developments in technology and society. The executive branch of government has usually privileged access to this type of knowledge, even if the capacities to absorb it may be limited. In the face of complexity, uncertainty and ambiguity of findings and interpretations, building and maintaining the necessary 'strategic intelligence' to ensure the strategic governance of technology and society is not an easy task. And even the attempt to have recourse to external advice that is contracted out to research and consulting organisations has a number of shortcomings, related in particular to the absorptive capacity and the accumulation of FTA knowledge needed to translate advice into political and administrative decision-making.

The situation in private firms has been very similar. Future intelligence units were increasingly expected to provide forward-looking knowledge for operational and $R \& D$ units rather than fulfilling an exploratory think-tank function for top-level management, but this development is currently being reconsidered in several firms (Daheim and Uerz 2008). This has led to a renewed interest in the institutionalisation of forward-looking intelligence, to ensure that the knowledge generated meets the needs and can be delivered in a timely manner on demand.

\subsubsection{Observation 4: Growing flexibility of FTA} systems. FTA in support of organisations' and institutions' embedding in decision-making is needed in the light of a fast-changing turbulent environment. Institutions are confronted with greater complexity and uncertainty in their context. The fast pace of technological change and the complexity of its societal repercussions make the interpretation of contextual developments very difficult. Organisations are facing major problems in identifying future challenges and providing solutions on time. A faster and continuous analysis and translation into actions of future challenges and opportunities is thus needed. This is particularly obvious when the focus extends beyond the challenges that societies face today and seeks to anticipate future challenges and transform them into opportunities. The need to deal with global issues implies a corresponding institutional capacity and the ability to respond at the appropriate international level.

Different models of FTA systems can be complementary in many respects. Service providers as well as FTA institutions need to be able to draw on networks for many purposes, and the boundaries between service provision and institutionalised forms of FTA are blurring. Exploring the balance between these three forms of FTA activities in empirical terms helps to improve our understanding of how effectively different combinations of activities work in their respective decision-making context and governance modes. Whether a specific model of FTA is appropriate for a transformative problem or not strongly depends on the wider institutional and organisational environment in which FTA is embedded, be it in the private or the public sector. Ultimately, an intelligent combination of FTA models needs to be put at the disposal of decision-makers, with projects and programmes providing targeted inputs, networking approaches delivering the 
most recent information on weak signals, and FTA institutions coordinating and integrating the different inputs in order to deliver advice quickly to policy-makers.

Thus, emerging forms of FTA facility are tending towards more embedded, internationally networked and multifunctional setups to respond to these challenges and address various needs both reactively and proactively. This form of combined FTA capacity includes elements of traditional, current and emerging rationales as identified in Table 3. The specific structures for realizing this range of FTA functions will vary and are likely to change in time, influenced by the needs and constraints imposed by novel developments such as the current economic and financial crisis. However, the coordination mode of governance that seems to be on rise in Europe - see for instance the debates on European Innovation Partnerships and Joint Programming - tends to favour such flexible arrangements for realizing FTA.

\section{Conclusions}

This paper has explored FTA responses to a highly dynamic environment where disruptive events highlight the fragility of governance systems. The transformations that such events lead to were categorised into four types: disruptive, ongoing, and by design, with the latter either triggered by policy shocks or managed as a smooth transition. In response to these systemic and structural transformations, FTA systems play a critical anticipatory and early warning capacity-building role, co-evolving with policy action in four archetypal modes of governance: integration, coordination, competition and co-existence. In turn three organisation models of FTA are identified: short-term projects and programmes, dedicated embedded FTA units, and networks of bundled facilities. Based on this, a framework of analysis was developed for tracing the evolution of FTA systems.

Empirical research highlights a diversity of FTA approaches and systems in practice, which reflect the different mixes of transformations, governance and organisational modes. The complementarity between models of FTA is apparent with service providers and FTA units drawing on networks, blurring the divide between the two. In general, the shift from short-term projects and programmes to institutionalised forms of FTA is due to the demanding policy environment and the need for more proactive approaches to address pressing global challenges. Institutions face greater complexity and difficulty in providing solutions on time, particularly when the policy focus extends beyond the challenges that societies face today, to anticipate future challenges and transform them into opportunities. This heralds a new generation of FTA systems, reflecting a stronger emphasis on flexibility, networking and institutionalisation, enabling delivery of a combination of different FTA instruments and allowing more customised responses to disruptive and transformative changes.

The question that arises from these observations on the possible future evolution of FTA is whether or not these types of developments will be sufficient to cope with the challenges of transformative and disruptive changes. As long as it is possible to anticipate the causes of any economic, social or environmental crisis, society is in a position to address them beforehand, either to deal with the likely consequences or even to transform them into opportunities. However, if the causes are not fully recognised or are recognised too late, crises are inevitable. Emerging shortages of food, water and other resources, due to demographic trends and human activities, will have far reaching economic and social consequences, and will thus become multilevel global challenges. Complex systems, like the global economy, entail emergent properties that can radically transform the system (e.g. Funtowitz and Ravetz 1994). While it is more and more difficult to anticipate when and how such changes will occur due to higher complexity, FTA can produce a better understanding of the overall landscape. By doing so, it can provide guidance on how to develop adaptive capabilities to enhance resilience and to exploit suddenly emerged opportunities.

The main problem is that governments and companies tend to deal with changes in a reactive rather than a proactive mode. Multiple factors influence the ways in which the future will evolve, and existing institutions have not yet been able to develop a fully systemic view of current and possible future situations to be prepared for properly shaping the future. Identifying weak signals and developing scenarios are crucial tools in preparing for the unexpected, thus enabling a clearer understanding of possible pathways to tackle the challenges, but this will not be enough. In addition, a periodic assessment of these scenarios would be necessary to update and adapt these in view of the latest world developments.

In this context, it is imperative that foresight initiatives to address global challenges are carried out at regular intervals to build a common understanding of current situations and to translate these into common visions of the future of the world which can be pursued jointly. Such initiatives can address and generate a range of impacts: building a continuous and shared approach to understand the present, exploring alternative futures and shaping the direction to follow, coupled with an evaluation of what has or has not been achieved from time to time to correct deviations and to continually adapt to new situations. Such initiatives would help to support evidence-based policy-making. Changing mindsets are needed in order to prepare and sensitise for using FTA in preparation of structural changes, and to prepare firms and governments accordingly. Scenario-based monitoring not only applies to the problems and challenges ahead, but also to the FTA systems that are already in place. There is a need 
to invest in iterative processes of assessment and monitoring in order to ensure that FTA systems can address fast-changing requirements of the future.

\section{Acknowledgements}

The views expressed are purely those of the author and may not in any circumstances be regarded as stating an official position of the European Commission.

\section{Notes}

1. This approach of drawing on the papers of the most recent FTA conference has been adopted, because the 2011 FTA Conference was explicitly dealing with disruptions and how to deal with them from the side of FTA. Due to the broad coverage of the conference, the contributions can be regarded as reflecting the current state of the debate. See $<\mathrm{http}$ ://foresight.jrc. ec.europa.eu/fta_2011/Programmeandpapers.htm > accessed 15 December 2011.

2. A f ifth possible way of social organisation would be the solitary person who escapes from coercive or manipulative social involvement altogether. However, this is not relevant for our abstraction.

3. Cf. FTA Conferences on <http://foresight.jrc.ec .europa.eu/fta.html> accessed 15 Dec 2011.

4. FARHORIZON Innovation Policy Workshop (Weber and Georghiou 2010) and ERAPRISM Policy Dialogue Brief on Innovation Policy (Georghiou and Harper 2010), <http://farhorizon.portals.mbs.ac.uk/> accessed 15 December 2011.

5. See for example the SESTI Project which uses Facebook and Twitter to identify and analyse weak signals (Amanatidou et al. 2012).

6. Europe 2020 Flagship Initiative Innovation Union, $<$ http://ec.europa.eu/research/innovation-union/pdf/ innovation-union-communication_en.pdf $>$ accessed 15 December 2011 (SEC 2010)

\section{References}

Ahlqvist, T., Valovirta, V. and Loikkanen, T. (2012) 'Innovation policy roadmapping as a systemic instrument for forward-looking policy design', Science and Public Policy, 39: 178-90.

—. (2011) 'Constructing systemic transformation capacities in a research and technology organisation: Applying diversified roadmap concept at VTT, Finland', Paper presented at the FTA 2011 Conference, held 12-13 May, Seville, Spain. $<$ http://foresight.jrc.ec.europa.eu/fta_2011/

Programmeandpapers.htm > accesse $\bar{d} 15$ Dec 2011.

Amanatidou, E. (2008) "Joint foresight': towards a mechanism for joint programming in Europe?", Foresight, 10: 103-17.

Amanatidou, E., Butter, M., Carabias, V., Könnölä, T., Leis, M., Saritas, O., Schaper-Rinkel, P. and van Rij, V. (2012) 'On concepts and methods in horizon scanning: Lessons from initiating policy dialogues on emerging issues', Science and Public Policy, 39: 208-21.

Brummer, V., Könnölä, T. and Salo, A. (2008) 'Foresight within Era-Nets: Experiences from the preparation of an international research programme', Technological Forecasting and Social Change, 75: 483-95.

Butter, M., Brandes, F., Keenan, M., Popper, R., Giesecke, S., Rijkers-Defrasne, S., Braun, A. and Crehan, P. (2009) 'Monitoring foresight activities in Europe and the rest of the world', Final Report of the European Foresight Monitoring Network EFMN. EUR 24043 EN. Brussels: European Commission.

Cachia, R., Compaño, R. and Da Costa, O. (2007) 'Grasping the potential of online social networks for foresight', Technological Forecasting \& Social Change, 74: 1179-203.

Cagnin, C., Amanatidou, E. and Keenan, M. (2012) 'Orienting European Innovation systems towards grand challenges and the roles that FTA can play', Science and Public Policy, 39: $140-52$.

Calof, J. (2012) 'Towards developing an instrument to evaluate Future Technology Analysis - A Canadian case study', Technological Forecasting and Social Change (forthcoming).

Calof, J., Miller, R. and Jackson, M. (2012) 'Towards impactful foresight: Viewpoints from Foresight consultants and academics', Foresight (forthcoming).

Cuhls, K. (2001) 'Foresight with Delphi surveys in Japan', Technology Analysis \& Strategic Management, 13: 555-69.

Daheim, C. and Uerz, G. (2008) 'Corporate foresight in Europe: From trend based logics to open foresight', Technology Analysis \& Strategic Management, 20: 321-36.

Dervin, B. (1998) 'Sense-making theory and practice: An overview of user interests in knowledge seeking and use', Journal of Knowledge Management, 2: 36-46.

European Commission. (2006) 'Creating an Innovative Europe', Report of the Independent Expert Group on R\&D and Innovation appointed following the Hampton Court Summit and chaired by Mr. Esko Aho, EUR 22005. Luxembourg: Office for Official Publications of the European Communities. <http://ec.europa.eu/invest-inresearch/pdf/download_en/aho_report.pdf> accessed 15 Dec 2011.

- (2008) 'Challenging Europe's Research - Rationales for the European Research Area (ERA)', Report of the ERA Expert Group, EUR 23326 EN. Luxembourg: Office for Official Publications of the European Communities. <http:// ec.europa.eu/research/era/pdf/eg7-era-rationales-final-report_ en.pdf $>$ accessed 15 Dec 2011.

European Research Area Board. (2009) 'Preparing Europe for a new Renaissance. A strategic view of the European Research Area', First Report of the European Research Area Board. Directorate-General for Research, EUR 23905 EN. Brussels: European Commission. <http://ec.europa.eu/research/erab/ pdf/erab-first-annual-report-06102009_en.pdf $>$ accessed 15 Dec 2011.

European Science Foundation. (2010) 'Contribution to Developing voluntary guidelines on framework conditions for joint programming in research - Foresight activities' (28 June 2010). Strasbourg: European Science Foundation.

Fernández Güell, J. M. and Redondo Gómez, L. (2012) 'Linking territorial foresight and urban planning', Foresight (forthcoming).

Funtowitz, S. and Ravetz, J. R. (1994) 'Emergent complex systems', Futures, 26: 568-82.

Geels, F. W. and Schot, J. (2007) 'Typology of sociotechnical transition pathways', Research Policy, 36: 399-417. 
Georghiou, L. and Cassingena Harper, J. (2010) 'Policy dialogue brief on innovation policy', Report from the ERAPRISM Project. <http://www.eraprism.eu/work packages.asp?SubFolder $=$ WP2 $>$ accessed 15 Dec 2011.

- (2011) 'From priority-setting to societal challenges in future-oriented technology analysis', Futures, 43: 229-31.

Joos, W., Carabias, V., Winistörfer, H. and Stücheli, A. (1999) 'Social aspects of public waste management in Switzerland', Waste Management, 19: 417-25.

Keenan, M., Cutler, P., Marks, J., Meylan, R., Smith, C. and Koivisto, E. (2012) 'Orienting international science co-operation to meet global grand challenges', Science and Public Policy, 39: 166-77.

Könnölä, T. and Haegeman, K. (2012b) 'Embedding foresight in transnational research programming', Science and Public Policy, 39: 191-207.

Könnölä, T., Carrillo Hermosilla, J., Loikkanen, T. and van der Have, R. (2009) Governance of energy system transition: Analytical framework and empirical cases in Europe and beyond, VTT Technical Research Centre of Finland Working Papers 134, 2009.

Könnölä, T., Salo, A., Cagnin, C., Carabias, V. and Vilkkumaa, E. (2012a) 'Facing the future: Scanning, synthesizing and sense-making in horizon scanning, Science and Public Policy, 39: 222-31.

Loveridge, D. and Street, P. (2005) 'Inclusive foresight', Foresight, 7: 31-47.

Nehme, C. C., de Miranda Santos, M., Fellows Filho, L. and Massari Coelho, G. (2012) 'Challenges in communicating the outcomes of a foresight study to advise decision-makers on policy and strategy', Science and Public Policy, 39: 245-57.

Nordmann, A. (2004) 'Converging technologies - Shaping the future of European societies', Report of an Expert Group to the European Commission. Brussels: European Commission. $<$ http://ec.europa.eu/research/conferences/2004/ntw/pdf/final_ report_en.pdf $>$ accessed 15 Dec 2011.

Rijkens-ǨKlomp, N. and van der Duin, P. (2012) Evaluating local and national public foresight studies from a user perspective, Futures (forthcoming).

Rotmans, J., Kemp, R. and van Asselt, M. (2001) 'More evolution than revolution: transition management in public policy', Foresight, 3: 15-31.
Salo, A. and Kuusi, O. (2001) 'Developments in parliamentary technology assessment in Finland', Science and Public Policy, 28: 453-64.

SEC. (2010) 'Commission Staff Working Document A rationale for action, accompanying the Europe 2020 Flagship Initiative Innovation Union', COM(2010) 546, pp. 9 and 89, <http://ec .europa.eu/research/innovation-union/pdf/rationale_en.pdf\# view $=$ fit $\&$ pagemode $=$ none $>$ accessed 15 Dec $201 \overline{1}$.

Skoufias, E. (2003) 'Economic crises and natural disasters: Coping strategies and policy implications', World Development, 31: 1087-102.

Taleb, N. N. (2007) The Black Swan: The Impact of Highly Improbable. New York: Random House.

Thompson, M., Ellis, R. and Wildavsky, A. (1990) Cultural Theory. Boulder, CO: Westview Press.

Tiits, M. and Kalvet, T. (2011) 'Foresight as an innovation policy tool in smaller catching-up economies: Grand narratives or intelligent piggybacking?', Paper presented at the FTA 2011 Conference held 12-13 May, Seville, Spain. $<$ http://foresight.jrc.ec.europa.eu/fta_2011/

Programmeandpapers.htm> accessed 15 Dec 2011.

Tukker, A. and Butter, M. (2007) 'Governance of sustainable transitions: about the 4(0) ways to change the world', Journal of Cleaner Production, 15: 94-103.

Van Rij, V. (2010) 'Joint horizon scanning: identifying common strategic choices and questions for knowledge', Science and Public Policy, 37: 7-18.

Warnke, P. (2011) 'Implementing systemic RTI priorities Recent experience from Germany', Paper presented at the FTA 2011 Conference, held 12-13 May, Seville, Spain. $<$ http://foresight.jrc.ec.europa.eu/fta_2011/

Programmeandpapers.htm> accessed 15 Dec 2011.

Weber, M. and Georghiou, L. (2010) 'Dynamising innovation policy: Giving innovation a central role in European policy', FarHorizon project report. < http://farhorizon.portals.mbs.ac .uk/Portals/73/docs/FarHorizon \% 20Dynamising \%20Inno vation \%20Policy.pdf $>$ accessed 15 Dec 2011.

Weber, M., Havas, A. and Schartinger, D. (2011) 'Exploring the potential impact of FLA on national innovation systems', Paper presented at the FTA 2011 Conference held 12-13 May, Seville, Spain. <http://foresight.jrc.ec.europa.eu/fta 2011/Programmeandpapers.htm > accessed 15 Dec 2011. 